hep-th/9312107

UCSBTH-93-39

\title{
Causal Properties of Free String Field Theory
}

\author{
David A. Lowe* \\ Department of Physics \\ University of California \\ Santa Barbara, CA 93106-9530
}

This paper examines the causal structure of the commutator of two string fields, in free light-cone string field theory. By treating the commutator as a distribution on infinite dimensional loop space, it is shown that the commutator vanishes when $\int d \sigma(\delta X(\sigma))^{2}<0$. Of more direct physical interest is the commutator of finite mass fields, obtained by smearing the string fields with appropriate wave functions. This is shown to vanish at spacelike separations, reproducing the usual point particle field theory result. The implications of this for the information spreading mechanism proposed by Susskind to solve the black hole information problem are discussed. Finally, it is verified that the above conclusions also hold for the superstring.

December, 1993

* lowe@tpau.physics.ucsb.edu 


\section{Introduction}

Until recently, the notion of causality in string theory has been relatively unexplored. One might expect some kind of Planck scale violation of causality due to the inherent nonlocal structure of strings moving in space-time. This question was addressed in [1] where the analog of the light-cone in string theory was considered in the framework of secondquantized bosonic light-cone string field theory [2 5]. In the second-quantized light-cone formalism one has a consistent operator formulation, so questions of local causality may be formulated in terms of the commutator of fields. Note that the string field in lightcone gauge only contains physical degrees of freedom, so, in principle, it is an observable. The calculations in [1] are valid when one introduces either a lattice cutoff on the string worldsheet or, equivalently, a cutoff on the number of string modes, although such a cutoff is not explicitly introduced in [1]. This is required to avoid divergences due to the infinite number of string states, which, as shown in [6] are sufficient to cause the propagator of point-like string states to blow up at spacelike separations, even in the superstring case. In this paper, we show this kind of cutoff is unnecessary, as long as one regards the commutator and the propagator as distributions on loop space. Then it is clear that to make well-defined statements one needs to smear these distributions with smooth test functions. This procedure leads to a simple proof of the vanishing of the commutator in the free theory at the string analog of spacelike separations. In [7] a weaker version of this condition was found, which appeared to allow for interference between string fields at spacelike separations.

To see what this causality condition means physically, it is important to consider not just eigenstates of the string localized in loop space, but finite mass wave packets. As is now well-known, these necessarily involve strings that extend over all space [8]. Nevertheless, we find these finite mass field operators commute at spacelike separations as is usual in point-particle quantum field theory. We argue that this implies a naïve interpretation of the information spreading mechanism recently suggested by Susskind to explain the black hole information problem, does not appear to occur, at least in flat-space, at the level of free string field theory.

The calculations discussed above were performed in bosonic string theory. In order to formulate a well-defined causal boundary in the first place, it was necessary to adjust the mass of the tachyon by hand, with the justification that in the full superstring field theory, the physics would be essentially the same. As a check on this we perform the calculation using second-quantized light-cone superstring field theory, where the string field lives on superspace. It is verified that the boundary of causal propagation is identical to that found in the bosonic string calculation.

\section{Causality and the Commutator in String Field Theory}

Here we will use the second quantized light-cone string field theory developed in [2 [5]. We introduce the light-cone coordinates

$$
X^{+}=\left(X^{0}+X^{d-1}\right) / \sqrt{2} \quad \text { and } \quad X^{-}=\left(X^{0}-X^{d-1}\right) / \sqrt{2} \text {. }
$$


Light-cone gauge corresponds to fixing $X^{+}(\sigma)=x^{+}$. The string field is then a function of the zero-modes $x^{+}, x_{0}^{-}$and the transverse coordinates $\vec{X}(\sigma)$. For simplicity we consider the case of open strings - the closed string case is very similar. The transverse coordinates are expanded as

$$
\vec{X}(\sigma)=\vec{x}_{0}+\sum_{l=1}^{\infty} 2 \vec{x}_{l} \cos (l \sigma) .
$$

The string field is decomposed in terms of creation and annihilation operators as

$$
\begin{aligned}
\Phi\left(x^{+}, x_{0}^{-}, \vec{X}(\sigma)\right) & =\int_{-\infty}^{\infty} \frac{d \vec{p}}{(2 \pi)^{d-2}} \int_{0}^{\infty} \frac{d p^{+}}{2 p^{+}} \sum_{\left\{\vec{n}_{l}\right\}}\left(A_{p^{+}, \vec{p},\left\{\vec{n}_{l}\right\}} e^{i\left(-p^{+} x_{0}^{-}-p^{-} x^{+}+\vec{p} \cdot \vec{x}_{0}\right)} \prod_{l=1}^{\infty} f_{\vec{n}_{l}}\left(\vec{x}_{l}\right)\right. \\
& + \text { h.c. }) .
\end{aligned}
$$

Here the $A$ operators obey canonical commutation relations

$$
\left[A_{p^{+}, \vec{p},\left\{\vec{n}_{l}\right\}}, A_{p^{+^{\prime}, \vec{p}^{\prime},\left\{\vec{n}_{l}{ }^{\prime}\right\}}}^{\dagger}\right]=2 p^{+} \delta\left(p^{+}-{p^{+}}^{\prime}\right)(2 \pi)^{d-2} \delta^{d-2}\left(\vec{p}-\vec{p}^{\prime}\right) \delta_{\left\{\vec{n}_{l}\right\},\left\{\vec{n}_{l}{ }^{\prime}\right\}},
$$

while the $f_{\vec{n}_{l}}\left(\vec{x}_{l}\right)$ are harmonic oscillator wave functions given by

$$
f_{\vec{n}_{l}}\left(\vec{x}_{l}\right)=\prod_{i=1}^{d-2} H_{n_{l}^{(i)}}\left(x_{l}^{(i)}\right) e^{-l x_{l}^{(i) 2}}
$$

with $H_{n_{l}^{(i)}}\left(x_{l}^{(i)}\right)$ a normalized Hermite polynomial. The light-cone energy is defined by

$$
p^{-}=\frac{\left(\vec{p}^{2}+m_{0}^{2}+\sum_{l=0}^{\infty} l \vec{n}_{l}\right)}{2 p^{+}} .
$$

The string propagator is then

$$
\begin{aligned}
& G\left(x_{1}^{+}, x_{1,0}^{-}, \vec{X}_{1}(\sigma) ; x_{2}^{+}, x_{2,0}^{-}, \vec{X}_{2}(\sigma)\right) \equiv\left\langle 0\left|\Phi\left(x_{1}^{+}, x_{1,0}^{-}, \vec{X}_{1}(\sigma)\right) \Phi\left(x_{2}^{+}, x_{2,0}^{-}, \vec{X}_{2}(\sigma)\right)\right| 0\right\rangle= \\
& \int_{0}^{\infty} \frac{d p^{+}}{2 p^{+}} e^{-i p^{+}\left(x_{1,0}^{-}-x_{2,0}^{-}\right)} K_{p^{+}}^{0}\left(x_{1}^{+}, \vec{x}_{1,0} ; x_{2}^{+}, \vec{x}_{2,0}\right) \prod_{l=1}^{\infty} K_{p^{+}}^{l}\left(x_{1}^{+}, \vec{x}_{1, l} ; x_{2}^{+}, \vec{x}_{2, l}\right),
\end{aligned}
$$

where we have defined

$$
\begin{aligned}
K_{p^{+}}^{0}\left(x^{+}, \vec{x} ; x^{+^{\prime}}, \vec{x}^{\prime}\right) & =\left(\frac{p^{+}}{2 \pi \delta x^{+}}\right)^{(d-2) / 2} \exp i\left(-\frac{m^{2}}{2 p^{+}} \delta x^{+}+\frac{p^{+}}{2 \delta x^{+}}(\delta \vec{x})^{2}\right), \\
K_{p^{+}}^{l}\left(x_{1}^{+}, \vec{x}_{1, l} ; x_{2}^{+}, \vec{x}_{2, l}\right) & =\left(\frac{2 l}{\pi\left(1-\exp \left(-2 i l \delta x^{+} / p^{+}\right)\right)}\right)^{(d-2) / 2} \\
& \times \exp \left(\frac{-i l}{\sin \left(l \delta x^{+} / p^{+}\right)}\left(\left(\left|\vec{x}_{1, l}\right|^{2}+\left|\vec{x}_{2, l}\right|^{2}\right) \cos \left(l \delta x^{+} / p^{+}\right)-2 \vec{x}_{1, l} \cdot \vec{x}_{2, l}\right)\right),
\end{aligned}
$$


with $\delta x^{+}=x_{1}^{+}-x_{2}^{+}$. The tachyon mass squared $m^{2}$ is to be treated as a positive adjustable parameter for the purpose of discussing the causal properties of the commutator. Of course, this is inconsistent with Lorentz invariance for the bosonic string. The superstring will be treated in section 4 , where $m^{2}=0$, to verify this procedure does indeed describe the correct physics.

It is important to regard the string field as an operator-valued distribution. This means the string field should, in general, be smeared with smooth test functions in order to obtain well-defined expressions. Likewise the propagator must also be regarded as a distribution. Now let us consider the commutator of two smeared string fields. For the purpose of seeing the causal properties of the commutator it suffices to leave a finite number $N-1$ of the string modes unsmeared. This leads to

$$
\begin{aligned}
& \int \prod_{l=N}^{\infty} d \vec{x}_{1, l} d \vec{x}_{2, l} g\left(\left\{\vec{x}_{1, l}\right\}\right) h\left(\left\{\vec{x}_{2, l}\right\}\right)\left[\Phi\left(X_{1}\right), \Phi\left(X_{2}\right)\right]= \\
& \quad \int \prod_{l=N}^{\infty} d \vec{x}_{1, l} d \vec{x}_{2, l} g\left(\left\{\vec{x}_{1, l}\right\}\right) h\left(\left\{\vec{x}_{2, l}\right\}\right)\left(G\left(X_{1} ; X_{2}\right)-G\left(X_{2} ; X_{1}\right)\right),
\end{aligned}
$$

where $g$ and $h$ are $C^{\infty}$, square integrable test functions. We wish to consider when we are allowed to rotate the $p^{+}$contour along either the positive or negative imaginary axis. We begin by considering the first term in (2.9), and we assume $\delta x^{+}>0$. Rotating the $p^{+}$integral along the positive imaginary axis leads to a convergent integral near $p^{+}=0$, however divergences may appear from the $p^{+} \rightarrow \infty$ limit. In this limit, this term in (2.9) looks like

$$
\begin{aligned}
& \int^{\infty} \frac{d p^{+}}{2 p^{+}} \int \prod_{l=N}^{\infty} d \vec{x}_{1, l} d \vec{x}_{2, l} g\left(\left\{\vec{x}_{1, l}\right\}\right) h\left(\left\{\vec{x}_{2, l}\right\}\right) e^{p^{+}\left(x_{1,0}^{-}-x_{2,0}^{-}\right)} \\
& \quad \times\left(\frac{p^{+}}{2 \pi \delta x^{+}}\right)^{(d-2) / 2} \exp \left(-\frac{p^{+}}{2 \delta x^{+}}\left(\delta \vec{x}_{0}\right)^{2}\right) \prod_{j=1}^{\infty}\left(\frac{p^{+}}{\pi \delta x^{+}}\right)^{(d-2) / 2} \exp \left(-\frac{p^{+}}{\delta x^{+}}\left(\delta \vec{x}_{j}\right)^{2}\right) .
\end{aligned}
$$

Note that in this limit the integrand is proportional to an infinite product of $\delta$-functions in $\delta \vec{x}_{j}$. Integrating over the test functions shows that this contribution to (2.9) is finite, provided

$$
2 \delta x_{0}^{-} \delta x^{+}-\delta \vec{x}_{0}^{2}-2 \sum_{l=1}^{N-1}\left|\delta \vec{x}_{l}\right|^{2}<0 .
$$

Likewise, the contour in the second term of (2.9) may be rotated along the negative imaginary axis provided (2.11) is satisfied. One then finds the two terms cancel and the commutator vanishes when (2.11) holds. We may now note this argument did not depend on the value of $N$, as long as it is finite, and did not depend on the precise nature of the test functions, as long as they were sufficiently smooth and square integrable. Therefore, formally taking the $N \rightarrow \infty$ limit, it is consistent to regard the commutator of two string fields as vanishing, in the sense of distributions, provided

$$
2 \delta x_{0}^{-} \delta x^{+}-\delta \vec{x}_{0}^{2}-2 \sum_{l=1}^{\infty}\left|\delta \vec{x}_{l}\right|^{2}<0
$$


This condition is stronger than that found in [7] which stated that the commutator vanishes when

$$
2 \delta x_{0}^{-} \delta x^{+}-\delta \vec{x}_{0}^{2}-2 \sum_{l=1}^{\infty}\left|\delta \vec{x}_{l}\right|^{2}<-1,
$$

which means that operators may interfere at the string analog of spacelike separations. This condition (2.13) appears inconsistent with the equal $x^{+}$canonical commutation relations of light-cone string field theory which require

$$
\left[\Phi(X), \Phi\left(X^{\prime}\right)\right]=\Delta\left(X(\sigma)-X^{\prime}(\sigma)\right),
$$

where $\Delta$ is a functional $\delta$-function. The source of the discrepancy lies in the treatment of the infinite number of modes of the string. In this paper we have smeared an infinite number of these modes with smooth test functions. When we do this, no matter what test functions we choose (as long as they satisfy the conditions described above) the interference at small spacelike separations found in [7] disappears. We conclude, therefore, that (2.12) gives the correct condition for the vanishing of the string field commutator, when this object is properly interpreted as a distribution on loop space.

\section{Physical Interpretation}

Let us see what the string light-cone condition (2.12) tells us when we consider finite mass wave packets of string fields. It is well known 8 that any finite mass superposition of string loops necessarily involves strings of infinite length. The field operator that creates, for example, a gauge boson, involves strings that occupy all of space. This is obtained by

$$
A^{k}\left(x^{+}, x_{0}^{-}, \vec{x}_{0}\right)=\int \prod_{l=1}^{\infty} d \vec{x}_{l} f_{\vec{n}_{l}}\left(\vec{x}_{l}\right) \Phi\left(x^{+}, x_{0}^{-}, \vec{x}_{0},\left\{\vec{x}_{l}\right\}\right)
$$

with $x_{0}$ (the center of mass of the particle) fixed, $\vec{n}_{l}=0$ for $l>1$ and $n_{1}^{k}=1$. Let us now ask when two gauge boson field operators commute. Applying (2.12) to each component of the $A$ field, one reproduces the usual field theory result that two gauge boson fields always commute at spacelike separations, i.e. when

$$
2 \delta x^{+} \delta x^{-}-\left|\delta \vec{x}_{0}\right|^{2}<0,
$$

and otherwise they may interferet. The same result holds for any finite mass string states. Note that the functions $f_{\vec{n}_{l}}\left(\vec{x}_{l}\right)$ are not smooth in the limit $l \rightarrow \infty$, as we required the test functions to be in the previous section. However, it may be verified by explicit calculation that relaxing this constraint does not lead to any problems in computing the commutator of two finite mass fields in the case at hand.

This would seem to indicate that the information contained in a finite mass wave packet is not spread over all space at the level of free string theory if observations are

$\dagger$ This result was developed in conversation with S. Giddings. 
performed at sufficiently small time resolutions, as recently suggested by Susskind [9], but propagates just as in ordinary point-particle field theory. To see this consider two observers $\mathcal{O}$ and $\mathcal{O}^{\prime}$ at spacelike separation. Here we will follow closely an argument made in the case of relativistic quantum field theory in [10]. We make the hypothesis that probability distributions may be computed using second-quantized string field theory. In addition, we assume that a measurement performed at $x_{0}^{\mu}$ corresponds to a measurement operator which is a function of finite mass field operators at $x_{0}^{\mu}$, and their derivatives. The corresponding projection operators will then also be local in the finite mass fields, and will commute at spacelike separations. Near $\mathcal{O}$ is the center of mass of some Planck mass string state (more precisely a localized wave packet of such states). $\mathcal{O}$ then performs a local field strength measurement which collapses the string state function. Now the question is, if $\mathcal{O}^{\prime}$ performs a local field strength measurement of sufficiently small time resolution, does she see the change in the information content of the system?

One interpretation of the results of [9] is that $\mathcal{O}^{\prime}$ should see the change in information, implying that superluminal communication between $\mathcal{O}$ and $\mathcal{O}^{\prime}$ is possible. However, because the projection operators always commute at spacelike separations, we see that $\mathcal{O}^{\prime}$ is unable to measure the influence of $\mathcal{O}$ on the state function. From this it seems the above interpretation of the effect conjectured in [9] does not occur, at least in flat Minkowski space, in free string theory.

Intuitively, this results from the fact that the wave function of a finite mass string excitation (for example, a superposition of excitations up to level $n$ ) has all the higher modes in their ground states. It is this infinite number of higher modes which cause the string wave function to spread over all space (in the sense that $\left\langle\left(\vec{X}(\sigma)-\vec{x}_{0}\right)^{2}\right\rangle$ diverges). However it is clear these modes do not carry information since they are in the same state for any finite mass excitation.

\section{Causality and the Commutator in Superstring Field Theory}

As a final check on the results of the previous sections, we consider the generalization to the superstring in ten flat space-time dimensions. In this theory, the worldsheet sweeps out a surface in superspace, parametrized by $X(\sigma, \tau)$ (the space-time coordinate) and two Majorana-Weyl Grassmann coordinates $\theta(\sigma, \tau)$ and $\tilde{\theta}(\sigma, \tau)$. We work in light-cone gauge where a consistent $\mathrm{SU}(4) \times \mathrm{U}(1)$ invariant superstring field theory was developed in [14] * This corresponds to the gauge choice

$$
\begin{aligned}
X^{+}(\sigma) & =x^{+}, \\
\gamma^{+} \theta & =\gamma^{+} \tilde{\theta}=0 .
\end{aligned}
$$

Here plus and minus refer to the longitudinal light-cone coordinates defined by

$$
V^{ \pm}=\left(V^{0} \pm V^{9}\right) / \sqrt{2} .
$$

* See [11,12] for earlier works on superstring field theory, and [13] for a recent review. 
In addition to the zero modes, the string wave function depends on the transverse $\mathrm{SO}(8)$ vector $X^{I}(\sigma, \tau)$ and the pair of $\mathrm{SO}(8)$ spinor coordinates $\tau^{a}(\sigma, \tau)$ and $\tilde{\theta}^{a}(\sigma, \tau)$. For chiral theories (type I and IIB) the $\theta$ 's are in the same spinor representation $\mathbf{8}_{S}$, while in the nonchiral theory (type IIA) we choose $\theta^{a}$ to be in $\boldsymbol{8}_{S}$ and $\tilde{\theta}^{a}$ to be in $\boldsymbol{8}_{C}$. In the $\mathrm{SU}(4) \times \mathrm{U}(1)$ formalism, the $\mathrm{SO}(8)$ spinor $\theta^{a}$ is decomposed into the $\mathrm{SU}(4)$ spinors $\theta^{\bar{A}}$ and $\lambda^{A}$, where $A=1,2,3,4$, and likewise for $\tilde{\theta}^{a}$.

For simplicity, let us restrict attention to the type I open string. Yang-Mills charges may be attached to the ends of the string for the groups $\mathrm{SO}(\mathrm{N})$ and $\operatorname{USp}(2 \mathrm{~N})$ as in [15]. The $\mathrm{SO}(32)$ theory is the only open-string theory free of anomalies at the quantum level. The string field belongs to the adjoint representation of the gauge group. The string field satisfies a non-orientability constraint

$$
\Phi^{a b}[X(\sigma), \theta(\sigma), \tilde{\theta}(\sigma)]=-\Phi^{b a}\left[X\left(2 \pi\left|p^{+}\right|-\sigma\right), \tilde{\theta}\left(2 \pi\left|p^{+}\right|-\sigma\right), \theta\left(2 \pi\left|p^{+}\right|-\sigma\right)\right]
$$

In the following we set $Z(\sigma)=(X(\sigma), \theta(\sigma), \tilde{\theta}(\sigma))$. The string field also satisfies a reality condition which requires the $\Phi$ field to be CTP self-conjugate. This means

$$
\hat{\Phi}^{a b}(X, \theta, \tilde{\theta})=\Phi^{a b *}\left(X, \theta / 4 p^{+}, \tilde{\theta} / 4 p^{+}\right)
$$

which, for example, restricts the field content of the massless open-string states to the super Yang-Mills multiplet. Here the symbol ^ denotes the functional Fourier transform with respect to the Grassmann variables

$$
\hat{\Phi}(X, \lambda, \tilde{\lambda})=\int D^{4} \theta D^{4} \tilde{\theta} e^{I(\lambda, \theta)} \Phi(X, \theta, \tilde{\theta})
$$

where we have defined

$$
I(\lambda, \theta)=\int_{0}^{2 \pi p^{+}} d \sigma\left(\lambda^{A}(\sigma) \theta^{\bar{A}}(\sigma)+\tilde{\lambda}^{A}(\sigma) \tilde{\theta}^{\bar{A}}(\sigma)\right) .
$$

From this point on we will suppress the group theory indices. The equal $x^{+}$commutation relations for the string fields satisfying (4.3) are

$$
\left[\Phi\left[Z_{1}(\sigma)\right], \Phi\left[Z_{2}(\sigma)\right]\right]=2 p_{2}^{+} \delta\left(p_{1}^{+}+p_{2}^{+}\right) \Delta^{16}\left[Z_{1}(\sigma)-Z_{2}(\sigma)\right] .
$$

Alternatively, one could relax (4.3) for the fields in the commutator, and subtract a piece proportional to

$$
\begin{aligned}
\Delta^{16}\left[Z_{1}(\sigma)-Z_{2}\left(2 \pi\left|p_{2}^{+}\right|-\sigma\right)\right] & =\Delta^{8}\left[X_{1}^{I}(\sigma)-X_{2}^{I}\left(2 \pi\left|p_{2}^{+}\right|-\sigma\right)\right] \\
& \times \Delta^{4}\left[\theta_{1}^{\bar{A}}(\sigma)-\tilde{\theta}_{2}^{\bar{A}}\left(2 \pi\left|p_{2}^{+}\right|-\sigma\right)\right] \Delta^{4}\left[\tilde{\theta}_{1}^{\bar{A}}(\sigma)-\theta_{2}^{\bar{A}}\left(2 \pi\left|p_{2}^{+}\right|-\sigma\right)\right]
\end{aligned}
$$


from the right-hand side of (4.7), as done in [11]. The free action for the string field in light-cone gauge is

$$
\begin{aligned}
S & =\int d x^{+} \int d^{16} Z \int_{0}^{\infty} \frac{d p^{+}}{2 p^{+}}-i \operatorname{tr}\left(\dot{\Phi}\left(Z,-p^{+}, x^{+}\right) \Phi\left(Z, p^{+}, x^{+}\right)\right) \\
& -\operatorname{tr}\left(\Phi\left(Z,-p^{+}, x^{+}\right) \int_{0}^{2 \pi p^{+}} d \sigma\left(-\pi\left(\frac{\delta}{\delta X}\right)^{2}+\frac{1}{\pi} X^{\prime 2}-2 i\left(\theta^{\prime} \frac{\delta}{\delta \theta}-\tilde{\theta}^{\prime} \frac{\delta}{\delta \tilde{\theta}}\right)\right) \Phi\left(Z, p^{+}, x^{+}\right)\right) .
\end{aligned}
$$

We impose the open string boundary conditions

$$
\begin{aligned}
X^{\prime}(0) & =X^{\prime}\left(2 \pi\left|p^{+}\right|\right)=0, \\
\theta^{\bar{A}}(0) & =\tilde{\theta}^{\bar{A}}(0), \\
\theta^{\bar{A}}\left(2 \pi\left|p^{+}\right|\right) & =\tilde{\theta}^{\bar{A}}\left(2 \pi\left|p^{+}\right|\right)
\end{aligned}
$$

and use the following modal decompositions

$$
\begin{aligned}
X(\sigma) & =x_{0}+\sum_{n=1}^{\infty} 2 x_{n} \cos \left(n \sigma / 2 p^{+}\right), \\
\theta^{\bar{A}}(\sigma) & =\frac{1}{2 \sqrt{2} p^{+}} \sum_{n=-\infty}^{\infty} R_{n}^{\bar{A}} e^{i n \sigma / 2 p^{+}} \\
\tilde{\theta}^{\bar{A}}(\sigma) & =\frac{1}{2 \sqrt{2} p^{+}} \sum_{n=-\infty}^{\infty} R_{n}^{\bar{A}} e^{-i n \sigma / 2 p^{+}}
\end{aligned}
$$

where the $R_{n}^{\bar{A}}$ should be regarded as Grassmann numbers. In terms of these modes, the Schrödinger equation for the string field is

$$
i \frac{\partial \Phi}{\partial x^{+}}=\left(\sum_{n=1}^{\infty}\left[\frac{n^{2} x_{n}^{2}}{p^{+}}-\frac{1}{4 p^{+}}\left(\frac{\delta}{\delta x_{n}}\right)^{2}\right]-\frac{1}{2 p^{+}}\left(\frac{\delta}{\delta x_{0}}\right)^{2}-\frac{1}{p^{+}} \sum_{m=-\infty}^{\infty} m R_{m}^{\bar{A}} \frac{\delta}{\delta R_{m}^{\bar{A}}}\right) \Phi .
$$

The propagator obtained from this equation is

$$
\begin{aligned}
G\left(Z_{1}, Z_{2}\right) & =\int_{0}^{\infty} \frac{d p^{+}}{2 p^{+}} K_{p^{+}}^{0}\left(\delta x^{+}, \delta x^{-}, \delta \vec{x}\right) \prod_{a=1}^{\infty} K_{p^{+}}^{a}\left(\delta x^{+}, \vec{x}_{1, a}, \vec{x}_{2, a}\right) \\
& \times \prod_{b=-\infty}^{\infty} L_{p^{+}}^{b}\left(\delta x^{+}, R_{1, b}, R_{2, b}\right) .
\end{aligned}
$$

Here $K_{p^{+}}^{0}$ is a free, mass $m$, particle propagator

$$
K_{p^{+}}^{0}\left(x^{+}, \vec{x} ; x^{+^{\prime}}, \vec{x}^{\prime}\right)=\left(\frac{p^{+}}{2 \pi \delta x^{+}}\right)^{(d-2) / 2} \exp i\left(-\frac{m^{2}}{2 p^{+}} \delta x^{+}+\frac{p^{+}}{2 \delta x^{+}}(\delta \vec{x})^{2}\right),
$$


the $K_{p^{+}}^{a}$ are bosonic harmonic oscillator propagators,

$$
\begin{aligned}
K_{p^{+}}^{a}\left(\delta x^{+}, \vec{x}_{1, a}, \vec{x}_{2, a}\right) & =\left(\frac{2 a}{\pi\left(1-\exp \left(-2 i a \delta x^{+} / p^{+}\right)\right)}\right)^{(d-2) / 2} \\
& \times \exp \left(\frac{-i a}{\sin \left(a \delta x^{+} / p^{+}\right)}\left(\left(\left|\vec{x}_{1, a}\right|^{2}+\left|\vec{x}_{2, a}\right|^{2}\right) \cos \left(a \delta x^{+} / p^{+}\right)-2 \vec{x}_{1, a} \cdot \vec{x}_{2, a}\right)\right)
\end{aligned}
$$

while the $L_{p^{+}}^{b}$ are a representation of the Fermi oscillator propagators

$$
\begin{aligned}
& L_{p^{+}}^{b}\left(\delta x^{+}, R_{1, b}, R_{2, b}\right)=R_{2, b} \exp \left(-i b \delta x^{+} / p^{+}\right)-R_{1, b} \quad \text { for } b \geq 0 \\
& L_{p^{+}}^{b}\left(\delta x^{+}, R_{1, b}, R_{2, b}\right)=R_{2, b}-\exp \left(-i|b| \delta x^{+} / p^{+}\right) R_{1, b} \quad \text { for } b<0 .
\end{aligned}
$$

In the superstring $m^{2}=0$, however to separate poles in the propagator we will take $m^{2}=0^{+}$.

Now we are ready to consider the commutator of the string field at different times

$$
\begin{aligned}
& {\left[\Phi\left[Z_{1}(\sigma), x_{1}^{+}\right], \Phi\left[Z_{2}(\sigma), x_{2}^{+}\right]\right]=} \\
& \int_{0}^{\infty} \frac{d p^{+}}{2 p^{+}}\left(K_{p^{+}}^{0}\left(\delta x^{+}, \delta x^{-}, \delta \vec{x}\right) \prod_{a=1}^{\infty} K_{p^{+}}^{a}\left(\delta x^{+}, \vec{x}_{1, a}, \vec{x}_{2, a}\right) \prod_{b=-\infty}^{\infty} L_{p^{+}}^{b}\left(\delta x^{+}, R_{1, b}, R_{2, b}\right)\right. \\
& \left.\quad-K_{p^{+}}^{0}\left(-\delta x^{+},-\delta x^{-},-\delta \vec{x}\right) \prod_{a=1}^{\infty} K_{p^{+}}^{a}\left(-\delta x^{+},-\vec{x}_{1, a},-\vec{x}_{2, a}\right) \prod_{b=-\infty}^{\infty} L_{p^{+}}^{b}\left(-\delta x^{+},-R_{1, b},-R_{2, b}\right)\right) .
\end{aligned}
$$

One may make the same kind of argument used above to determine when the commutator of two string fields vanishes. Again it is essential to regard the commutator as a distribution- this time on super-loop space $Z(\sigma)$. When smeared by smooth functions the Grassmann factors make no contribution to the convergence in the $p^{+} \rightarrow \infty$ limit- the convergence is dominated only by the factors depending on the bosonic coordinates $\vec{x}_{a}$ as before. One therefore reaches the same conclusion: the commutator of two string fields vanishes if

$$
2 \delta x^{+} \delta x^{-}-\left|\delta \vec{x}_{0}\right|^{2}-2 \sum_{a=1}^{\infty}\left|\delta \vec{x}_{a}\right|^{2}<0 .
$$

Note the natural space-time Lorentz covariant form of this condition is

$$
\int d \sigma(\delta X(\sigma))^{2}<0
$$

\section{Conclusions}

In this paper we have carefully reconsidered the derivation of the light-cone condition on string loop space, first considered in [1]. By properly treating the string commutator as 
a distribution on loop space we have shown that the light-cone condition originally found in [1] is correct, which is a stronger condition than that found in a later calculation [7]. These calculations were performed in free string field theory. An interesting open question is whether interactions, due to their non-local nature in string theory, will change this light-cone condition in the full theory. $\dagger$

Applying this causal condition to finite mass field operators, it was shown that the usual point-particle quantum field theory light-cone condition is recovered. It was argued that the information spreading mechanism proposed in [9] to solve the black hole information paradox, does not occur at the level of free string field theory.

As a final check on the consistency of the above calculations, the causal condition was formulated in the framework of light-cone superstring field theory. The bosonic string contains a tachyon, whose mass must be set to zero by hand in the analogous bosonic calculations. Therefore, any completely consistent discussion of causality should be made in the context of the superstring. It was shown, nevertheless, the same causal condition is found.

Acknowledgments: The author wishes to thank S. Chaudhuri, S. Giddings, J. Polchinski, T. Samols and A. Strominger for helpful discussions, and M. Green for pointing out reference [6]. This work is supported in part by NSF grant PHY91-16964.

$\dagger$ Note added: In fact, this now appears to be the case [16]. 


\section{References}

[1] E. Martinec, "The Light Cone in String Theory," Class. Quant. Grav. 10 (1993) L187.

[2] S. Mandelstam, Nucl. Phys. B64 (1973) 205; Nucl. Phys. B83 (1974) 413; Phys. Rep. 13C (1974) 259.

[3] E. Cremmer and J.-L. Gervais, Nucl. Phys. B76 (1974) 209; Nucl. Phys. B90 (1975) 410.

[4] M. Kaku and K. Kikkawa, Phys. Rev. D10 (1974) 1110; Phys. Rev. D10 (1974) 1823.

[5] J.F.L. Hopkinson, R.W. Tucker and P.A. Collins, Phys. Rev. D12 (1975) 1653.

[6] E. Corrigan and D.B. Fairlie, Nucl. Phys. B91 (1975) 527; M.B. Green, Nucl. Phys. B124 (1977) 461; Phys. Lett. B266 (1991) 325; Phys. Lett. B302 (1993) 29; preprint QMW-91-02.

[7] E. Martinec, "Strings and Causality," hepth/9311129, EFI-93-65.

[8] M. Karliner, I. Klebanov and L. Susskind, "Size and Shape of Strings," Int. Jour. Mod. Phys. A3 (1988) 1981.

[9] L. Susskind, Stanford preprint SU-ITP-93-18, July 1993; SU-ITP-93-21, August 1993; Rutgers preprint RU-93-44, September 1993.

[10] P.H. Eberhard and R.R. Ross, Found. Phys. Lett. 2 (1989) 127.

[11] M.B. Green and J.H. Schwarz, Nucl. Phys. B218 (1983) 43.

[12] M.B. Green, J.H. Schwarz and L. Brink, Nucl. Phys. B219 (1983) 437.

[13] A. Restuccia and J.G. Taylor, Phys. Rep. 174 (1989) 283.

[14] M.B. Green and J.H. Schwarz, Nucl. Phys. B243 (1984) 475.

[15] J.E. Paton and H.M. Chan, Nucl. Phys. B10 (1969) 516.

[16] D.A. Lowe, L. Susskind, J. Uglum, in preparation. 